\title{
The sound of black holes fusing
}

\author{
Reflections on the decibel lead Joe Wolfe and Oleg Sushkov to considerations of infinite waveguides and to why we \\ didn't hear the LIGO black-hole merger.
}

$\mathrm{N}$ o one uses deciseconds or decivolts, so why are sound levels given in decibels? The bel, named for Alexander Graham Bell, is a measure of the sound intensity level as $\log _{10}\left(I / I_{\text {ref }}\right)$, where $I$ is intensity and $I_{\text {ref }}$ a reference intensity. One reason for using the ten-times-smaller unit comes from telephony in the 1920s: conveniently, the decibel roughly equals the signal attenuation along a mile of standard cable.

The intensity level in decibels $(\mathrm{dB})$ is $10 \log _{10}\left(I / I_{\text {ref }}\right)$. For sound, the usual reference intensity is $1 \mathrm{pW} \mathrm{m}^{-2}$. Because sound pressure $p$ can be measured more easily than sound intensity, sound level measurements are usually, in fact, sound pressure levels. The intensity equals the sound pressure squared, $p^{2}$, divided by the specific acoustic impedance, $z$. For this reason, the sound pressure level is given by $20 \log _{10}\left(p / p_{\text {ref }}\right)$. Here, the commonly used reference $p_{\text {ref }}$ is $20 \mu \mathrm{Pa}$, which corresponds approximately to $1 \mathrm{pW} \mathrm{m}^{-2}$ in air under normal conditions. So doubling the wave intensity or power adds $3 \mathrm{~dB}$ to the level, whereas doubling the pressure increases the level by $6 \mathrm{~dB}$.

Acoustic impedances are often inferred by comparison with that of a known duct. But where to find a pure, frequencyindependent acoustic resistance? One answer is an acoustically infinite waveguide - such as those installed in the ceiling space of our physics building at UNSW Sydney. For sound, an effectively infinite waveguide need not be very long. For a $200 \mathrm{~m}$ pipe, the first echo takes more than a second to return, and for most acoustic frequencies, that's enough time for a measurement.

When it comes to sound, it's convenient that $1 \mathrm{~dB}$ is comparable with the justnoticeable difference ${ }^{2}$, meaning it's hard to notice differences less than $1 \mathrm{~dB}-$ as these sound files demonstrate ${ }^{3}$. A sound level of $0 \mathrm{~dB}$ (that is, pressure of $20 \mu \mathrm{Pa}$ or intensity $1 \mathrm{pW} \mathrm{m}^{-2}$ ) is close to the threshold for human hearing at a frequency $f$ of $1 \mathrm{kHz}$. Yes, hearing is that sensitive - but it's also frequency dependent. This dependence is included in the definition of the phon using standardized hearing response curves, and setting the level in phons equal to the pressure level in $\mathrm{dB}$ for a $1 \mathrm{kHz}$ pure tone. But the sound level in phons or $\mathrm{dB}$ is not proportional to loudness. The complicated relation between perceived loudness and intensity is better approximated by a power law than a log relation. For levels above 40 phons, loudness measured in sones is judged to double for each increase by 10 phons, and 1 sone is set equal 40 phons.

Let's get quantitative: at a distance $r$ of $1 \mathrm{~m}$ from an isotropic sound source with power $P$ of $1 \mathrm{~mW}$, the intensity is simply the power divided by the area of a sphere of radius $r$, yielding $\sim 0.1 \mathrm{~mW} \mathrm{~m}^{-2}$ or $80 \mathrm{~dB}$. For something more exotic, let's look at the first black-hole merger observed by $\mathrm{LIGO}^{4}$. In the last milliseconds before the merger, gravitational waves with a power of $3.6 \times 10^{49} \mathrm{~W}$ were detected from a distance of $410 \mathrm{Mpc}$. A similar naive calculation gives an intensity of around $20 \mathrm{~mW} \mathrm{~m}^{-2}$.

Now $20 \mathrm{~mW} \mathrm{~m}^{-2}$ is not small - it's more intense than moonlight. A sound wave with the same intensity would have a level of $103 \mathrm{~dB}$ at frequencies around $100 \mathrm{~Hz}$, which is within our hearing range. So, why didn't we hear the black-hole merger? Well, a sound wave with $20 \mathrm{~mW} \mathrm{~m}^{-2}$ produces a strain $\epsilon$ of about $2 \times 10^{-5}$ in air whereas the black-hole merger produced strains in space of $\sim 10^{-21}$ and thus deformations in the ear of order $10^{-23} \mathrm{~m}$. Further, while sound waves in air produce isotropic strains, gravitational waves are quadrupolar.

Gravity (a gauge wave) and sound (a Goldstone wave) are qualitatively different types of wave. Expressed independently of frequency or wavelength, sound intensity goes as strain squared $\sqrt{\kappa^{3} / \rho} \epsilon^{2}$, where $\kappa$ is the adiabatic modulus and $\rho$ the density. In contrast, the gravitational wave intensity goes as the square of the derivative of the strain: $\left(c^{3} / 8 \pi G\right)(\dot{\epsilon})^{2}$ (resulting in $\left.\left(\pi c^{3} / 2 G\right) f^{2} \epsilon^{2}\right)$, where $c$ is the speed of light and $G$ is Newton's constant.

The dependence of the intensity on $c$ and $G$ tells us that gravitational strains have small gradients. But fundamentally, we can't compare these different 'media' since different objects have different accelerations in an electric field, so electrical forces cannot be described with geometrical theories analogous to general relativity. Finally, although sound is a classical wave, $\kappa$ and $\rho$ of matter depend ultimately on particles and their quantum-mechanical interactions, whereas general relativity is universal and - at least to our current knowledge unrelated to quantum mechanics.

\section{Joe Wolfe and Oleg P. Sushkov} UNSW Sydney, Sydney, New South Wales, Australia.

e-mail:j.wolfe@unsw.edu.au; sushkov@unsw.edu.au

Published online: 4 November 2019

https://doi.org/10.1038/s41567-019-0686-2

References

1. Dickens, P., Smith, J. R. \& Wolfe, J. J. Acoust. Soc. Am. 121, 1471-1481 (2007)

2. Johnson, J. H., Turner, C. W., Zwislocki, J. J. \& Margolis, R. H. J. Acoust. Soc. Am. 93, 983-991 (1993).

3. Wolfe, J. dB: What is a decibel? Physclips https://www.animations physics.unsw.edu.au/jw/dB.htm (UNSW, 2005)

4. Abbott, B. P. et al. (LIGO Scientific Collaboration and Virgo Collaboration). Phys. Rev. Lett. 116, 061102 (2016).

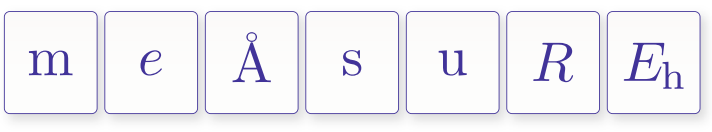

$\mu_{0} \varepsilon_{0} \alpha \mathrm{R} \mathrm{R}$

